13

\title{
Автоэмиссионная шахматная структура на основе алмазографитовых кластеров
}

\author{
(C) М.В. Давидович, ${ }^{1,2}$ P.К. Яфаров ${ }^{1,2}$ \\ ${ }^{1}$ Саратовский национальный исследовательский государственный университет им. Н.Г. Чернышевского, \\ 410012 Саратов, Россия \\ ${ }^{2}$ Саратовский филлиал ИРЭ РАН им. В.А. Котельникова, \\ 410054 Саратов, Россия \\ e-mail: davidovichmv@info.sgu.ru
}

(Поступило в Редакцию 7 июня 2017 г.)

Предложены и рассчитаны вакуумная автоэмиссионная триодная структура с напыленными в шахматном порядке на проводящую подложку (катод) высокоомными полупроводниковыми или диэлектрическими прямоугольными параллелепипедами микронных размеров, а также структура с наноразмерной пленкой на катоде из напыленных алмазографитовых кластеров. Теоретически и экспериментально показано, что рассмотренные структуры обладают существенно большими эмиссионными способностями по сравнению с непокрытым катодом.

DOI: 10.21883/JTF.2018.02.45422.2375

\section{Введение}

В последнее время большое значение уделяется изучению эмиссионных свойств различных углеродных структур, особенно в связи с необходимостью создания сильноточных автоэмиссионных источников электронных пучков для ЛБВ, плоских панелей и для других приложений [1-6]. Известные автоэмиссионные структуры острийного и лезвийного типов не удовлетворяют ряду требований [1-2]. В острийных автокатодах эмиссия идет в основном с острий, разогрев которых приводит к взрывной эмиссии и не позволяет получать большие интегральные значения тока и долговечные катоды. Матричные автокатоды создают большой разброс электронов по углам и не позволяют получать широкие и тонкие ленточные пучки. Лезвийные автокатоды не позволяют получать большие токи.

В настоящей работе предложено использовать рельефные углеродные структуры из алмазных и графитовых микроструктур из нанокластеров на длинном плоском катоде с большой площадью (рис. 1). Шахматные микроструктуры, состоящие из алмазографитовых нанокластеров (рис. 2,a), мы получали микроволновым плазмохимическим осаждением из паров этанола $[7,8]$. Алмазная фаза соответствует высокоомному полупроводниковому состоянию, тогда как графитовая фаза имеет проводимость, близкую к металлической. Такие плоские структуры демонстрируют увеличение эмиссии на 2-3 порядка $[9,10]$ по сравнению с традиционной теорией Фаулера-Нордгейма, хорошо работающей для металлов. Экспериментально исследованы разные автоэмиссионные структуры с квазиплоской поверхностью, состоящей из различных структур углерода: фулеренов, нанотрубок, графеновых чешуек и лент, стеклоуглерода, алмазных и графитовых кластеров [1,2,5-10]. Повышенная полевая эмиссия наблюдается для всех алло- тропных модификациях углерода, что делает их весьма перспективными для создания сильноточных источников электронных пучков. В работах $[9,10]$ показано, что повышенная эмиссия не связана с усилением поля за счет острийного эффекта, что позволяет получать токоотбор практически со всей поверхности эмиттера без существенного разогрева каких-либо ее элементов и без образования взрывной эмиссии. Это также позволяет увеличить интегральный ток и долговечность автокатодов при меньших напряжениях. Оценки по формуле Фаулера-Нордгейма для таких структур дают физически неоправданные значения работы выхода (РВ) порядка $0.01-0.1 \mathrm{eV}$, что явно не соответствует квантовомеханическим расчетам и экспериментам для углерода в виде графита $(4.6-5 \mathrm{eV})$ и других его структур типа фулеренов, нанотрубок, графена, РВ которых имеет такой же порядок. Соответственно при нахождении таких структур на поверхности графита или металла РВ не должна существенно изменяться, но это не так. Различные аллотропные модификации углерода, находящиеся на поверхности катода, демонстрируют феноменальную эмиссию. Увеличение эмиссии, которой формально соответствует понижение РВ до порядков долей $\mathrm{eV}$, до сих пор строго не объяснено. Обзор возможных теорий дан в [11], где также предложена теория проникновения поля в полупроводниковый материал соответствующей углеродной фазы эмиттера. В работе [10] указанный эффект объяснен резонансным туннелированием в результате размерного квантования при образовании уровней размерного квантования, совпадающих с уровнем Ферми. В работах [12-19] применен классический подход на основе многократных электрических изображений и вычисления профиля барьера, а усиление эмиссии объяснено на основе размерного эффекта уменьшения толщины потенциального барьера примерно на толщину пленки, а также и его высоты. 


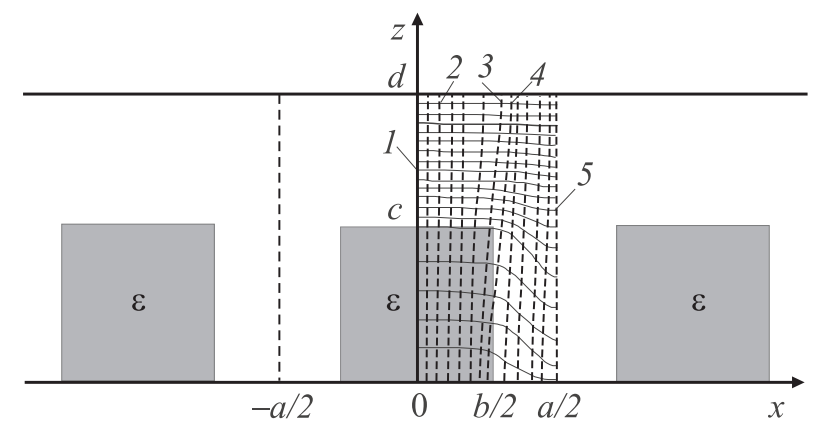

Рис. 1. Шахматная эмиссионная структура с периодически расположенными диэлектрическими блоками: сплошными линиями показаны эквипотенциали, штриховыми линиями силовые линии.

Пленка из аллотропных модификаций углерода (без учета пористости) имеет диэлектрическую проницаемость (ДП) порядка 16, что существенно уменьшает силы изображений при переходе в нее электронов из металла. Указанное значение соответствует алмазу и графиту. Реально следует учесть структуру и провести гомогенизацию. Например, массивный образец стеклоуглерода обладает повышенной эмиссионной способностью за счет пористости, эффекта проникновения поля и большого числа поверхностных состояний. На рассматриваемых структурах экспериментально получена плотность тока порядка $1000 \mathrm{~A} / \mathrm{cm}^{2}$.

Рассмотрим вакуумную диодную структуру рис. 1 с двумерно-периодическими диэлектрическими прямоугольными параллелепипедами с размерами $b \times b \times c$ и ДП $\varepsilon$, расположенными на проводящем катоде с периодом $a$ по осям $x$ и $y$. Потенциал на катоде $U_{c}$. Потенциал на аноде $U_{a}$. Расстояние между катодом и анодом $d$. Целью работы является построение траекторий туннелирования электронов с катода на анод, определение потенциальных барьеров вдоль траекторий и получение усредненной по траекториям и энергиям плотности автоэмиссионного тока структуры. Задача решается в несколько этапов. Первой задачей является нахождение распределения периодического потенциала в структуре. Второй задачей является нахождение потенциала для точечного заряда $q$, расположенного в структуре и удовлетворяющего уравнению Пуассона. Такой потенциал не симметричен. В силу симметрии удобно рассматривать четыре симметрично расположенных по $x$ и $y$ заряда $q$ в периоде структуры, одновременно и синхронно туннелирующих с катода. Каждый из зарядов практически не создает поля вдоль направления движения остальных и не изменяет потенциальный барьер для них, а поперечные силы их расталкивания для электронов ничтожны. Мы пренебрегаем пространственным зарядом, что вполне оправдано при автоэлектронной эмиссии почти до предельно достижимых плотностей тока [1], но выбираем из пучка четыре симметрично расположенных в ячейке периодичности электрона, считая, что плотность тока не является критически малой. Это позволяет сохранить условие периодичности. Соответственно решаем уравнение

$$
\nabla \nabla\left[\varepsilon(\mathbf{r}) U\left(\mathbf{r}, \mathbf{r}^{\prime}\right)\right]=-\left(q / \varepsilon_{0}\right) \sum_{i=1}^{4} \delta\left(\mathbf{r}-\mathbf{r}^{\prime}\right),
$$

где вектор $\mathbf{r}_{1}^{\prime}$ имеет координаты $\left(x^{\prime}, y^{\prime}, z^{\prime}\right)$, дельтафункция является двумерно-периодической, а индексам 2, 3, 4 соответствуют инверсии $x^{\prime} \rightarrow-x^{\prime}, y^{\prime} \rightarrow-y^{\prime}$ и $x^{\prime} \rightarrow-x^{\prime}, y^{\prime} \rightarrow-y^{\prime}$. Решение (1) с точностью до множителя $q / \varepsilon_{0}$ есть периодическая функция Грина $(\Phi Г)$ уравнения Пуассона: $G\left(\mathbf{r}-\mathbf{r}^{\prime}\right)=\left(\varepsilon_{0} / q\right) U\left(\mathbf{r}, \mathbf{r}^{\prime}\right)$. Поскольку ДП является ступенчатой функцией, (1) пишем в виде

$$
\nabla^{2} U(\mathbf{r})=-q \tilde{\delta}\left(\mathbf{r}-\mathbf{r}^{\prime}\right) /\left(\varepsilon_{0} \varepsilon\right),
$$

где в области вакуума $\varepsilon=1$. В (2) зависимость от координат зарядов опущена, а тильда означает сумму дельтафункций из (1). Однако (2) не справедливо на границе диэлектрика: там имеют место связанные поверхностные заряды, обусловленные скачком нормальной компоненты поля. Их плотность обозначим $\sigma$. Соответственно эти источники надо учесть в (2). Третьей задачей является нахождение потенциальной энергии электрона $V(\mathbf{r})$, перемещаемого с поверхности катода из точки $\mathbf{r}^{\prime}$ в некоторую точку $\mathbf{r}$ структуры. Эта потенциальная энергия определяет форму потенциального барьера в задаче туннелирования электрона с катода на анод. Четвертая задача состоит в том, чтобы определить форму траектории электрона, вылетающего нормально к поверхности катода со скоростью Ферми и двигающегося на анод, и вычислить коэффициент туннелирования вдоль такой траектории. Пятой задачей является нахождение интегрального тока по всем траекториям вылета с учетом распределения электронов по энергиям. Далее для электрона полагаем $q=-e$.

\section{1. Определение периодического потенциала}

Решение уравнений (1), (2) можно получить как общее решение уравнения Лапласа и частное решение уравнения Пуассона. Симметричное решение уравнения Лапласа вне диэлектрика в области $z>c$ ищем в виде

$$
\begin{aligned}
U(\mathbf{r})= & U_{A} z / d+\sum_{m=0}^{\infty} \sum_{n=0}^{\infty} a_{m n}^{+} \cos \left(k_{m} x\right) \cos \left(k_{n} y\right) \\
& \times \sinh \left(\kappa_{m n}(z-d)\right),
\end{aligned}
$$

где $k_{m}=2 m \pi / a, k_{n}=2 n \pi / a, \kappa_{m n}=\sqrt{k_{m}^{2}+k_{n}^{2}}$. Оно удовлетворяет граничному условию на аноде. Вне диэлектрика при $z<c$ решение ищем в виде

$$
U(\mathbf{r})=U_{A} z / q+\sum_{m=0}^{\infty} \sum_{n=0}^{\infty} b_{m n}^{+} \cos \left(k_{m} x\right) \cos \left(k_{n} y\right) \sinh \left(\kappa_{m n} z\right)
$$


Оно удовлетворяет граничному условию на катоде. Задаем потенциал внутри диэлектрика

$$
\begin{aligned}
& U(\mathbf{r})=\sum_{m=0}^{\infty} \sum_{n=0}^{\infty} \cos \left(\tilde{k}_{m} x\right) \cos \left(\tilde{k}_{n} y\right)\left[a_{m n}^{-} \sinh \left(\tilde{\kappa}_{m n} z\right)\right. \\
& \left.+b_{m n}^{-}\left(1-\cosh \left(\tilde{\kappa}_{m n} z\right)\right)\right]+\sum_{m=0}^{\infty} \sum_{n=1}^{\infty} \sin \left(\bar{k}_{n} z\right) \\
& \times\left[c_{m n}^{-} \cos \left(\breve{k}_{m} x\right) \cosh \left(\chi_{m n} y\right)+d_{m n}^{-} \cos \left(\breve{k}_{m} y\right) \cosh \left(\chi_{m n} x\right)\right] \\
& +\sum_{m=0}^{\infty} \sum_{n=1}^{\infty}(1-\cos (n \pi z / c))\left[f_{m n}^{-} \cos \left(\breve{k}_{m} x\right) \cosh \left(\chi_{m n} y\right)\right. \\
& \left.+g_{m n}^{-} \cos \left(\breve{k}_{m} y\right) \cosh \left(\chi_{m n} x\right)\right] .
\end{aligned}
$$

Он также удовлетворяет граничному условию на катоде. Здесь $\tilde{k}_{m}=\breve{k}_{m}=2 m \pi / b, \tilde{\kappa}_{m n}=\sqrt{\tilde{k}_{m}^{2}+\tilde{k}_{n}^{2}}, \bar{k}_{n}=n \pi / c$, $\chi_{m n}=-\sqrt{\breve{k}_{m}^{2}+\bar{k}_{n}^{2}}$. Сравнивая (3) и (4), имеем $a_{m n}^{+} \times$ $\times \sinh \left(\kappa_{m n}(c-d)\right)=b_{m n}^{+} \sinh \left(\kappa_{m n} c\right)$. Кроме того, должны выполняться соотношения $a_{m n}^{+} \cosh \left(\kappa_{m n}(c-d)\right)=$ $=b_{m n}^{+} \cosh \left(\kappa_{m n} c\right)$. При больших индексах эти соотношения эквивалентны, а при небольших эквивалентны приближенно. При нулевых индексах имеем $a_{00}^{+}=b_{00}^{+}$. Кроме того, потенциал должен быть непрерывным на границе диэлектрика, а его нормальная производная терпеть скачок, равный $\varepsilon$. Из этих условий можно выразить коэффициенты в (5) через $a_{m n}^{+}$и получить решение в виде рядов. Полагая, например, $z=c$, имеем два условия $U(x, y, z-0)=U(x, y, z+0)$ и $\varepsilon(\partial / \partial z) U(x, y, z-0)=(\partial / \partial z) U(x, y, z+0)$, из которых определяем $a_{m n}^{-}$и $b_{m n}^{-}$. Полагая $x=b$, имеем два условия $U(x-0, y, z)=U(x+0, y, z)$ и $\varepsilon(\partial / \partial x) U(x-0, y, z)=(\partial / \partial z) U(x+0, y, z+0)$, из которых выражаем $d_{m n}^{-}$и $g_{m n}^{-}$. Из условий при $y=b$ выражаем коэффициенты $c_{m n}^{-}$и $f_{m n}^{-}$. Получается бесконечная система линейных алгебраических уравнений с бесконечным числом неизвестных. Ее решаем методом усечения. Для этого задаем несколько коэффициентов $a_{m n}^{+}$и выражаем через них все остальные. Для решения используем метод итераций с нулевыми начальными коэффициентами в правых частях итерационных формул.

\section{2. Метод изображений для плоского диода}

Указанный выше подход можно использовать для определения поля в присутствии одного точечного заряда, считая, что он не сильно изменяет в среднем потенциал в структуре. Однако точечный заряд сильно изменяет поле вблизи него самого, делая его сингулярным, и это изменение следует учитывать для получения потенциальной энергии электрона. Будем решать уравнение Пуассона с одним точечным зарядом методом изображений [16-18]. Сначала рассмотрим заряд $q$ в бесконечном плоском вакуумном диоде без диэлектрика. Пусть заряд находится в точке $\rho^{\prime}=0, z^{\prime}$ цилиндрической системы координат. Решение задачи методом бесконечных изображений имеет вид $[13,14]$

$$
\begin{aligned}
U\left(\rho, z, z^{\prime}\right)= & \frac{q}{4 \pi \varepsilon_{0}} \sum_{n=-\infty}^{\infty}\left[\frac{1}{R\left(\rho, z-z^{\prime}-2 n d\right)}\right. \\
& \left.-\frac{1}{R\left(\rho, z+z^{\prime}-2 n d\right)}\right] .
\end{aligned}
$$

Здесь $R(\rho, z)=\sqrt{\rho^{2}+z^{2}}$. Потенциал (6) сингулярный в точке нахождения заряда $\rho=0, z=z^{\prime}$. Используя его, можно найти потенциальную энергию электрона при перемещении его с катода вдоль нормали в точку $z$. Для этого следует положить $\rho=0, q=-e$, выбросить из (6) сингулярный член $\left|z-z^{\prime}\right|^{-1}$ и положить $z=z^{\prime}$. Имеем

$$
\begin{aligned}
& U\left(0, z, z^{\prime}\right)=\frac{1}{4 \pi \varepsilon_{0}}\left[\frac{1}{\left|z-z^{\prime}\right|}-\frac{1}{z+z^{\prime}}\right. \\
& \left.\quad+\sum_{n=1}^{\infty}\left(\frac{4 n d}{(2 n d)^{2}-\left(z^{\prime}-z\right)^{2}}-\frac{4 n d}{(2 n d)^{2}-\left(z^{\prime}+z\right)^{2}}\right)\right] .
\end{aligned}
$$

Удаляя сингулярный член, тем самым мы находим потенциал от изображений заряда $U^{\prime}(z)$, действующий в точке его расположения $z$ [20]:

$$
U^{\prime}(0, z)=-\frac{q}{8 \pi \varepsilon_{0}}\left[\frac{1}{z}+2 \sum_{n=1}^{\infty}\left(\frac{n d}{(n d)^{2}-z^{2}}-\frac{1}{n d}\right)\right]
$$

Сам заряд, естественно, на себя не действует. На электрон действует сила $-e E_{z}(z)$, где $E_{z}(z)=$ $=-d U^{\prime}(0, z) / d z$. Определяем работу по перемещению электрона. Если электрон перемещается на $d z$ от катода, то расстояние между ним и изображениями изменяется на $2 d z$. Поэтому потенциальная энергия имеет вид $[20,21]$

$$
W_{p}=-\frac{e^{2}}{16 \pi \varepsilon_{0}}\left[\frac{1}{z}+2 \sum_{n=1}^{\infty}\left(\frac{n d}{(n d)^{2}-z^{2}}-\frac{1}{n d}\right)\right] .
$$

Возникновение двойки в знаменателе можно объяснить еще и тем, что на заряд вблизи металлического экрана действует сила, пропорциональная квадрату удвоенного расстояния до экрана. Однако формула (8) дает бесконечную отрицательную потенциальную энергию электрона на катоде. Это имеет место из-за того, что сила зеркального изображения бесконечна при нулевом расстоянии. На самом же деле эта сила перестает действовать на расстоянии порядка половины постоянной решетки кристалла, т.е. на расстоянии, когда электронные оболочки перекрываются. Обозначим это расстояние $\delta$. Это значение тесно связывается с РВ. Аналогично вблизи анода притягивающая к нему сила 

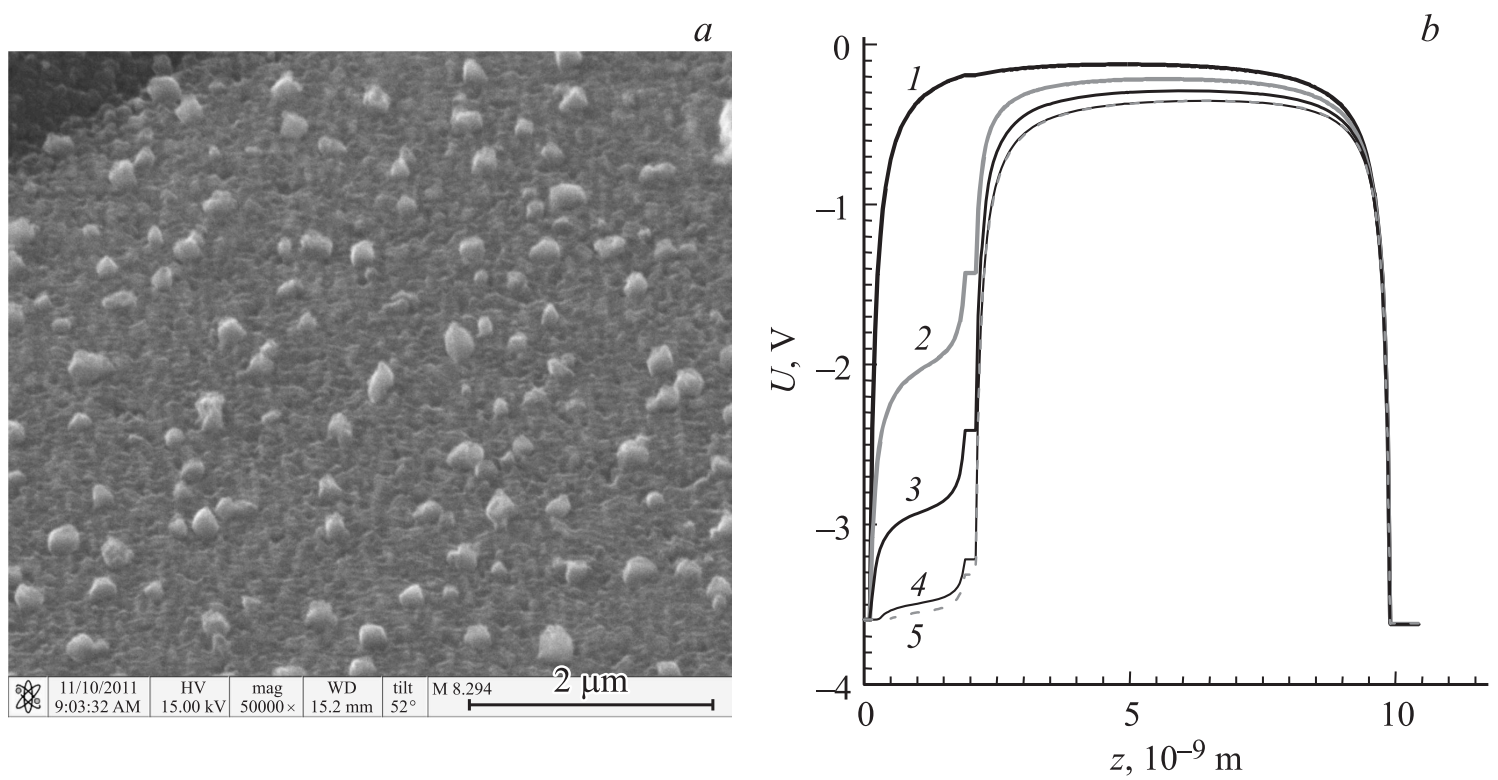

Рис. 2. СЭМ изображение нанокомпозитной алмазографитовой пленочной структуры $(a) ; b-$ распределение потенциала $U$ в промежутке катод-анод $(d=10 \mathrm{~nm})$ без анодного напряжения при наличии пленки толщины $t=2 \mathrm{~nm}$ для значений ее диэлектрической проницаемости $\varepsilon: 2$ (кривая 2); 4 (3); 10 (4); 12 (5), 1 - без пленки.

перестает действовать на расстояниях меньше $\delta$. Поэтому потенциальную энергию можно записать так

$$
\begin{aligned}
W_{p}(z)= & -\frac{e^{2}}{16 \pi \varepsilon_{0}}\left[\frac{1}{z+\delta}-\frac{2}{d}+\frac{1}{d+\delta-z}+\frac{1}{d+z}\right. \\
& \left.+\frac{2 z^{2}}{d^{3}} \sum_{n=2}^{\infty} \frac{1}{\left(n^{2}-(z / d)^{2}\right) n}\right] .
\end{aligned}
$$

Входящий в (9) ряд весьма быстро сходится и имеет значения $\xi(3)-1=0.202$ при $z=0, \quad 8 \ln (2)-$ $-16 / 3$ при $z=d / 2$ и $1 / 4$ при $z=d$. Очевидно, функция (9) симметричная относительно центра диода и $W_{p}(0)=W_{p}(d)=-e^{2} /\left(16 \pi \varepsilon_{0} \delta\right)$. В центре структуры при $z=d / 2$ из (8), (9) имеем

$W_{p}(d / 2)=-\left(4 \pi \varepsilon_{0} d\right)^{-1} e \sum_{n=1}^{\infty}(-1)^{n} n^{-1}=-e \ln (2) /\left(4 \pi \varepsilon_{0} d\right)$.

Полагая потенциальную энергию на катоде равной энергии Ферми, отсчитываемой от дна зоны проводимости $W_{p}(0)=E_{F}-E_{0}$, получаем $\mathrm{PB}$ из полубесконечного образца $W_{0}=e^{2} /\left(16 \pi \varepsilon_{0} \delta\right)$. Заметим, что для металлов, отдающих один электрон в зону проводимости, эта формула хорошо коррелирует с потенциалом ионизации иона $+1 e$, если за $\delta$ взять радиус его электронной оболочки. Здесь глубина зоны проводимости есть $E_{0}=E_{F}+W_{0}$. Во всех остальных членах мы положили $\delta=0$, поскольку $\delta \ll d$. При большом расстоянии катод-анод $W_{p}(d / 2) \approx 0$ и $\mathrm{PB} W \approx W_{0}$. В общем случае РВ связана с введенным параметром равенством

$$
W=W_{p}(d / 2)-W_{p}(0)=W_{0}(1-2.772 \delta / d) .
$$

Это та работа, которую нужно совершить, чтобы электрон достиг максимумы барьера и перешел на анод. Соотношения получены при нулевом напряжении на аноде. Образованный потенциальный барьер в отсутствие пленки широкий и симметричный относительно центра (рис. 2, b, кривая 1). Наличие пленки, как показано далее, приводит к несимметричному барьеру. Наличие напряжения на аноде приводит к образованию узкого несимметричного потенциального барьера (рис. 3). Для этого к (9) добавляем энергию $-e U_{a} z / d$. В ряде (9) ограничимся одним членом, что означает учет нескольких ближайших изображений. Следует отметить, что ряд в (9) быстро сходится. В результате получим весьма точную формулу, оставив в сумме один член и умножив на множитель 3:

$$
\begin{aligned}
W_{p}(z)= & -\frac{e^{2}}{16 \pi \varepsilon_{0}}\left[\frac{1}{z+\delta}+\frac{2 z^{2}-2 z \delta-d \delta}{d(d+\delta-z)(d+z)}\right. \\
& \left.+\frac{6 z^{2}}{2 d\left(4 d^{2}-z^{2}\right)}\right]
\end{aligned}
$$

Эта функция симметричная: $W_{p}(0)=W_{p}(d)=E_{F}$. В центре она равна $W_{p}(d / 2)=-2.533 e^{2} /\left(16 \pi \varepsilon_{0} d\right)$, что отличается от точного значения на $8 \%$. Учет нескольких членов ряда в (9) позволяет получить точность не хуже $1 \%$, однако проще использовать в (11) корректирующий множитель $1+1.08 \sin (\pi x / d)$. Таким образом, потенциальный барьер в плоском вакуумном диоде определяется функцией $V(z)=-e^{2} U_{a} z / d+W_{p}(z)$. 


\section{3. Метод изображений для плоского диода с диэлектрическим слоем}

В работах [13-15] методом электрических изображений найдена потенциальная энергия электрона в плоском вакуумном диоде с диэлектрической пленкой на катоде. Показано, что если ДП пленки достаточно большая (порядка 10 и более), потенциальный барьер сужается, а его высота уменьшается. Эффект наиболее сильный, если толщина пленки порядка ширины потенциального барьера в ее отсутствие, при этом промежуток $d$ катод-анод составляет 2-3 толщины пленки $c$. Эффект можно объяснить тем, что поле в диэлектрической пленке ослабляется в $\varepsilon$ раз, за счет чего ослабляются силы электрических изображений (на границе металл-пленка возникает двойной электрический слой). Электроны легко туннелируют в диэлектрик, из которого идет туннелирование в вакуум. На границе диэлектрик-вакуум также возникают силы изображений, но более слабые при большей напряженности поля: в случае большой ДП практически все анодное напряжение приложено к вакуумному промежутку пленка-анод. На границе катод-пленка барьера практически нет, а более узкий и низкий барьер возникает на границе диэлектрическая пленка-вакуум (рис. 3). Приведенные результаты получены с использованием метода изображений относительно трех границ: двух металлических при $z=0, z=d$ и одной диэлектрической при $z=c$. Заряд $q$ относительно диэлектрического полупространства имеет изображение

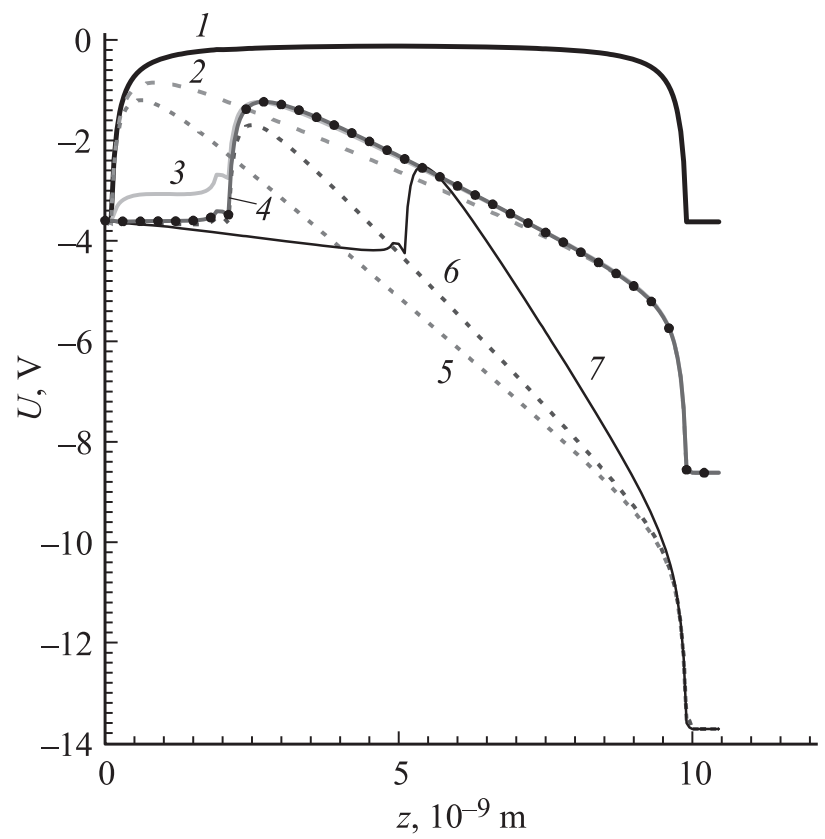

Рис. 3. Распределение потенциала при разных напряжениях на аноде без пленки (кривые 1,2,5), для пленок толщины $t=2 \mathrm{~nm}(3,4,6)$ и пленки с $t=5 \mathrm{~nm}(7): V_{A}=0 \mathrm{~V}(1)$; $U_{a}=5 \mathrm{~V} \quad(2-4) ; \quad U_{A}=10 \mathrm{~V} \quad(5-7) ; \varepsilon=4 \quad(3) ; \varepsilon=10 \quad$ (7); $\varepsilon=12(4,6)$. $q^{\prime}=k d[13,22]$, где $k=(1-\varepsilon) /(1+\varepsilon)-$ коэффициент отражения потока вектора электростатической индукции. Можно также получить решение указанной задачи, используя метод изображений для двух диэлектрических границ при $z= \pm c$ и одной металлической на аноде при $z=d$. Но при этом надо использовать два противоположных заряда, симметрично расположенных относительно катода $z=0$ и создающих на нем электрическую стенку. В случае пленки решение усложняется: имеется две области (внутри и вне пленки), где имеются разные представления потенциала. Вблизи границы с диэлектриком силы изображений также перестают действовать, и можно ввести размер, связанный с РВ из диэлектрика. По-видимому, этот параметр больше, а PB из диэлектрического образца меньше, что можно объяснить проникновением поля в диэлектрик и меньшей концентрацией свободных носителей в нем. Под диэлектриком здесь можно понимать и высокоомные полупроводниковые пенки. В рассматриваемом случае также можно получить простые и достаточно точные формулы [2], описывающие форму потенциального барьера. В настоящей работе мы, однако, рассмотрим другой более простой метод, основанный на том, что поле внутри структуры определяется зарядом $q$ и связанной поверхностной плотностью заряда $\sigma$ на границе диэлектрика и вакуума. В случае отсутствия заряда потенциал можно представить в виде

$$
\begin{aligned}
& U(z)= \\
& = \begin{cases}U_{a}(z) /[\varepsilon d-(\varepsilon-1) c], & 0 \leq z \leq c, \\
U_{a}(\varepsilon z-c(\varepsilon-1)) /[\varepsilon d-(\varepsilon-1) c], & c<z \leq d .\end{cases}
\end{aligned}
$$

Этому потенциалу соответствует плотность связанного поверхностного заряда при $z=c: \sigma_{-}=E_{d} \varepsilon_{0}(\varepsilon-1)$, где $E_{d}=-U_{a} /[\varepsilon d-(\varepsilon-1) c]$ - электрическое поле в диэлектрике. Противоположная плотность $\sigma_{+}=$ $=-E_{d} \varepsilon_{0}(\varepsilon-1)$ на пленке должна возникнуть при $z=0$. Полная плотность связанного поверхностного заряда при $z=0$ равна $\sigma=E_{d} \varepsilon_{0}$. Она включает в себя также плотность $\sigma_{0}^{-}=E_{d} \varepsilon_{0} \varepsilon$, наведенную на металле катода. Такая же положительная (с противоположным знаком) плотность наводится на аноде: $\sigma_{0}^{+}=-E_{d} \varepsilon_{0}$. Таким образом $\sigma=\sigma_{0}^{-}+\sigma_{+}=\sigma_{0}^{-}-\sigma_{-}$, т.е. пленка уменьшает связанный заряд на катоде. Емкость соответствующего конденсатора на единицу площади есть $C=\sigma_{0}^{+} / U_{a}=\varepsilon_{0} \varepsilon /[\varepsilon(d-c)+c]$. Заметим, что концепция связанного поверхностного заряда для однородного диэлектрика позволяет исключить диэлектрик из рассмотрения и анализировать поля зарядов в вакууме.

Теперь рассмотрим заряд $q$ над диэлектрической пластиной в точке $z^{\prime}=c+h$ при отсутствии анодного напряжения $U_{A}=0$. Он создает изображение $-q^{\prime}=-k q$ в точке $z^{\prime}=c-h$, которым можно ограничиться в случае большой толщины пластины $(c \gg h)$. Для строгого учета обоих электродов достаточно построить потенциал этих 
двух зарядов на основе приведенных выше формул, например (11). Этот потенциал действует в области $z>c$. В области $z<c$ заряд воспринимается как его изображение $q^{\prime \prime}=2 \varepsilon q /(\varepsilon+1)=(1-k) q$ в точке его расположения. При этом считается, что все пространство как бы заполнено диэлектриком. Если же заряд находится в диэлектрике, то в нем он воспринимается как эффективный заряд $q^{\prime}=q / \varepsilon$ (поле ослабляется в $\varepsilon$ раз за счет образования вокруг заряда двойного электрического слоя). Вне диэлектрика тогда он воспринимается как заряд $2 q /(\varepsilon(\varepsilon+1))$.

\section{4. Метод функций Грина}

Фиктивные заряды - удобный метод описания поля. Их действие эквивалентно поверхностным зарядам на границе диэлектрика. Мы будем использовать метод связанных поверхностных зарядов. Если $G\left(\mathbf{r}, \mathbf{r}^{\prime}\right)-$ функция Грина потенциала точечного заряда в пустом плоском конденсаторе, то потенциал точечного заряда в присутствии диэлектрика есть

$$
U(\mathbf{r})=\frac{q}{\varepsilon_{0} \varepsilon((\mathbf{r}))} G\left(\mathbf{r}, \mathbf{r}^{\prime}\right)+\frac{1}{\varepsilon_{0}} \oint_{S} G\left(\mathbf{r}, \mathbf{r}^{\prime}\right) \sigma\left(\mathbf{r}^{\prime}\right) d^{2} r .
$$

Если точка наблюдения в (13) находится в области диэлектрика, где $\varepsilon(\mathbf{r})>1$, то это фактически означает использование заряда $q^{\prime}=q / \varepsilon$. Поэтому основная задача - получение поверхностной плотности $\sigma$ на границе диэлектрика. ФГ в (13) имеет вид $G\left(\mathbf{r}, \mathbf{r}^{\prime}\right)=$ $=\varepsilon_{0} U\left(\rho, z, z^{\prime}\right), \quad$ где $\quad$ в $\quad(6)$ следует взять $\rho=$ $=\sqrt{\left(x-x^{\prime}\right)^{2}+\left(y-y^{\prime}\right)^{2}}$ и положить $q=1$. В случае периодической структуры удобно предположить, что заряды $q$ также расположены периодически. Тогда периодическая ФГ $\tilde{G}\left(\mathbf{r}, \mathbf{r}^{\prime}\right)$ строится периодическим продолжением:

$$
\tilde{G}\left(\mathbf{r}, \mathbf{r}^{\prime}\right)=\sum_{m=-\infty}^{\infty} \sum_{n=-\infty}^{\infty} G\left(\mathbf{r}, x^{\prime}+m a, y^{\prime}+n a, z^{\prime}\right)
$$

Кроме такого координатного представления возможно и спектральное представление периодической ФГ:

$$
\begin{gathered}
\tilde{G}\left(\mathbf{r}, \mathbf{r}^{\prime}\right)=\frac{1}{a^{2}} \sum_{m=-\infty}^{\infty} \sum_{n=-\infty}^{\infty} \exp \left(\frac{2 i m\left(x-x^{\prime}\right)}{a}\right. \\
\left.+\frac{2 i m\left(y-y^{\prime}\right)}{a}\right) \frac{g_{m n}\left(z, z^{\prime}\right)}{\kappa_{m n}}, \\
g_{m n}\left(z, z^{\prime}\right)=\frac{-1}{\sinh \left(\kappa_{m n} d\right)} \\
\times \begin{cases}\sinh \left(\kappa_{m n} z\right) \sinh \left(\kappa_{m n}\left(z^{\prime}-d\right)\right), & z<z^{\prime}, \\
\sinh \left(\kappa_{m n} z^{\prime}\right) \sinh \left(\kappa_{m n}(z-d)\right), & z>z^{\prime} .\end{cases}
\end{gathered}
$$

Она комплексная. Для вычислений с действительным потенциалом следует использовать ее реальную или мнимую часть. В случае симметричного по двум координатам потенциала удобна ФГ

$$
\begin{aligned}
\tilde{G}\left(\mathbf{r}, \mathbf{r}^{\prime}\right)= & \frac{1}{a^{2}} \sum_{m=-\infty}^{\infty} \sum_{n=-\infty}^{\infty} \cos \left(\frac{2 m x}{a}\right) \cos \left(\frac{2 m x^{\prime}}{a}\right) \\
& \times \cos \left(\frac{2 n y}{a}\right) \cos \left(\frac{2 n y^{\prime}}{a}\right) \frac{g_{m n}\left(z, z^{\prime}\right)}{\kappa_{m n}} .
\end{aligned}
$$

Нетрудно видеть, что эти ФГ удовлетворяют уравнению $\nabla^{2} \tilde{G}\left(\mathbf{r}, \mathbf{r}^{\prime}\right)=-\delta\left(\mathbf{r}-\mathbf{r}^{\prime}\right)$ с периодической объемной дельта-функцией. Действительно, при $\mathbf{r} \neq \mathbf{r}^{\prime}$ ФГ удовлетворяют уравнению Лапласа. При $\mathbf{r} \neq \mathbf{r}^{\prime}$ первая производная по $\mathrm{z}$ имеет единичный скачок, а при втором дифференцировании возникает дельта-функция. ФГ (17) также можно получить методом сшивания. Фактически (17) есть формула суммирования Пуассона для (15).

Метод ФГ позволяет записать решение задачи с зарядом $q$ в точке $\mathbf{r}_{0}$ в виде

$$
U(\mathbf{r})=q G\left(\mathbf{r}, \mathbf{r}_{0}\right)+U_{0}(\mathbf{r})+\frac{1}{\varepsilon_{0}} \oint_{S} G\left(\mathbf{r}, \mathbf{r}^{\prime}\right) \sigma\left(\mathbf{r}^{\prime}\right) d^{2} r .
$$

Здесь $U_{a}(\mathbf{r})=U_{a} z / d-$ потенциал, создаваемый анодом в пустой структуре, $\sigma(\mathbf{r})$ - поверхностный заряд, создаваемый этим потенциалом и зарядом на границе диэлектрика. Вместо $G$ в случае четной периодической ФГ под интегралом в (18) следует использовать ФГ (17), а внеинтегральный член взять в виде

$$
\begin{aligned}
q\left[G\left(\mathbf{r}, x_{0}, y_{0}, z\right)\right. & +G\left(\mathbf{r},-x_{0}, y_{0}, z\right)+G\left(\mathbf{r}, x_{0},-y_{0}, z\right) \\
& \left.+G\left(\mathbf{r},-x_{0},-y_{0}, z\right)\right] .
\end{aligned}
$$

Это означает движение симметрично расположенных четырех зарядов к аноду. Особенность уравнения (18) в том, что в силу (17) часть поверхности диэлектрика, лежащая на катоде, не создает поле. Его не создает также заряд на катоде и на аноде. Уравнение (18) превращается в интегральное уравнение для определения $\sigma(\mathbf{r})$, если наложить граничные условия на потенциал:

$$
\mathbf{v}(\mathbf{r})\left[\varepsilon \frac{\partial}{\partial v} U(\mathbf{r}-0)-\frac{\partial}{\partial v} U(\mathbf{r}+0)\right]_{r \in S}=0 .
$$

Для решения (19) удобно представить поверхностную плотность заряда в виде $\sigma(\mathbf{r})=\sigma_{0}(\mathbf{r})+\sigma_{q}(\mathbf{r})$, где $\sigma_{0}(\mathbf{r})$ - поверхностная плотность в отсутствие заряда, $\sigma_{q}(\mathbf{r})$ - плотность, индуцируемая зарядом. Для определения плотности, индуцированной зарядом, будем рассматривать один заряд над диэлектрической полуплоскостью. Это приближение хорошо работает, когда размеры диэлектрической структуры порядка и более толщины образующегося потенциального барьера. При сближении зарядов к центру плотность заряда возрастает и стремится к учетверенному значению. Но РВ не должна зависеть от точки вылета электрона. Следует рассматривать одноэлектронное туннелирование. Энергетически 
оно наиболее выгодно, следовательно, его вероятность максимальная. Поэтому форму потенциального барьера определяем исходя из (18), но с симметричной ФГ (17), считая, что один электрон не нарушает симметричность потенциала. Решая интегральное уравнение (18) с ФГ для вакуума для границы раздела вакуумполупространство, найдем $\sigma_{q}(\mathbf{r}): \sigma_{q}(\rho)=k q /\left(\rho^{2}+z_{0}^{2}\right)$. Заряд расположен в вакууме на оси $z$ на высоте $z_{0}$ над диэлектрическим полупространством. Этот же результат следует из метода изображений. Практически удобно использовать только плотность заряда $\sigma_{0}(\mathbf{r})$, а вместо $\sigma_{q}(\mathbf{r})$ брать изображение для электрона, положив $q=-e$.

\section{5. Эквипотенциали, формы потенциальных барьеров, ток эмиссии}

На рис. 1 представлены линии эквипотенциалей и силовые линии структуры с $d=19, a=19, b=10$, $c=11 \mathrm{~nm}$ и ДП $\varepsilon=15$ при анодном напряжении $U_{a}=10 \mathrm{~V}$. В первом приближении можно считать, что один электрон не изменяет потенциал и движется вдоль какой-либо эквипотенциали. На самом деле в силу инерционности электрона это не так. Однако если скорость электрона близка по направлению к касательной к силовой линии, это имеет место. Для этого при классическом подходе импульс электрона не должен быть слишком большим в местах максимальной кривизны силовых линий. Это следует относить к части траектории после прохождения барьера, где движение можно считать классическим. Строго говоря, для построения траекторий следует решать уравнения квантовой механики при наличии у электрона внутри металла импульса Ферми, который направлен под некоторым углом к границе. Решить такую одночастичную задачу даже для простой плоской границы и определить РВ и вероятность туннелирования до сих пор не удалось (см., например, [23]), и наиболее продуктивным методом до сих пор является метод изображений $[2,20]$. Как альтернативный можно отметить метод функционала плотности [24]. Мы используем сведение трехмерной задачи туннелирования к одномерным задачам туннелирования вдоль силовых линий поля с определением профилей потенциальных барьеров вдоль каждой из линий. Для определения тока можно считать, что электроны вылетают с катода нормально и равномерно, и определять коэффициенты туннелирования $D_{i}(E)$ по каждой из траекторий. Здесь энергия электрона, налетающего на границу катода, лежит в интервале $-E_{0}<E<-E_{0}+E_{F}$ от дна зоны проводимости $-E_{0}$ до энергии Ферми. Тогда плотность прямого тока в районе траектории $i$ без учета температуры $(T=0)$ имеет вид [1]

$$
J_{i}^{+}=e \int_{-E_{0}}^{-E_{0}+E_{F}} n_{z}(E) D_{i}(E) d E .
$$

Здесь $n_{z}(E)$ - число электронов, налетающих в секунду на границу катода и имеющих нормальную компоненту импульса $p_{z}=\sqrt{2 m E}$. В этом интеграле удобно сделать замену переменных $E^{\prime}=E+E_{0}$ и интегрировать от нуля до энергии Ферми. Для учета влияния малой температуры интеграл можно распространить до энергии $E_{m}=E_{F}+k T$. В общем случае следует взять бесконечный верхний предел. Следует также учесть обратный ток. С классической точки зрения движение электронов с анода вдоль силовых линий локально энергетически наименее выгодно. Поскольку мы ограничились одномерным рассмотрением задачи, считаем тем не менее, что обратному туннелированию соответствуют те же профили барьеров. Тогда полная плотность тока есть $J_{i}=J_{i}^{+}-J_{i}^{-}$. Для полной плотности тока в районе траектории $i$ имеем [20]

$$
J_{i}=\int_{0}^{E_{m}} D_{i}(\eta) \xi(\eta) d \eta
$$

Здесь при $T=0$ следует взять $\xi(\eta)=e U$ при $0<\eta<$ $<E_{F}-e U_{a}, \xi(\eta)=E_{F}-\eta$ при $E_{F}-e U_{a}<\eta<E_{F}$ и $\xi(\eta)=0$ при $\eta>E_{F}$. При этом $E_{m}=E_{F}=\mu$. При $k T \ll E_{F}$ следует взять $E_{m}=\mu+k T$ и учитывать размытость при вычислении $\xi$ :

$$
\zeta=\frac{4 \pi m e}{h^{3}} \int_{0}^{\infty}\left[f(E)-f\left(E-e U_{a}\right)\right] p_{\rho} d p_{\rho} .
$$

Интегрирование в (22) идет по поперечным компонентам импульса. При получении (21) предположено $D_{i}^{+}=D_{i}^{-}=D_{i}$, т.е. обратное туннелирование имеет место при той же энергии электрона, при этом следует использовать функции распределения $f(E)=[1+\exp ((E-\mu) / \beta)+1]^{-1}$ на катоде и $f\left(E+e U_{a}\right)$ на аноде, $\beta=k T$. Полный ток получается суммированием по траекториям. Если считать, что электрон рассеивается на аноде при туннелировании и его избыточная энергия диссипируется, то следует использовать разные коэффициенты туннелирования $D_{i}^{+}$ и $D_{i}^{-}$с учетом распределения электронов по энергиям, отличающиеся тем, что энергия всех электронов на аноде меньше на величину $-e U_{A}$. В этом случае барьер при обратном туннелировании больше, а функции распределения следует взять одинаковыми. Для плотности туннельного тока пишем $J_{i}=J_{i}^{+}-J_{i}^{-}=e\left(N_{i}^{+}-N_{i}^{-}\right)$, где

$$
N_{i}^{ \pm}=\frac{4 \pi m}{h^{3}} \int_{E_{0}^{ \pm}}^{E_{m}^{ \pm}} D_{i}^{ \pm}\left(E_{z}\right)\left(\int_{0}^{\infty} f(E) d E_{\rho}\right) d E_{z} .
$$

Здесь $E_{0}^{+}=E_{0}, E_{0}^{-}=E_{0}-e U_{a}, E_{m}^{-}=E_{m}^{+}-e U_{a}, E=$ $=E_{\rho}+E_{z}$. При $T=0$ следует взять $E_{m}^{+}=E_{F}$. Тогда интеграл от функции распределения в (23) равен $E_{F}-E_{z}$. 

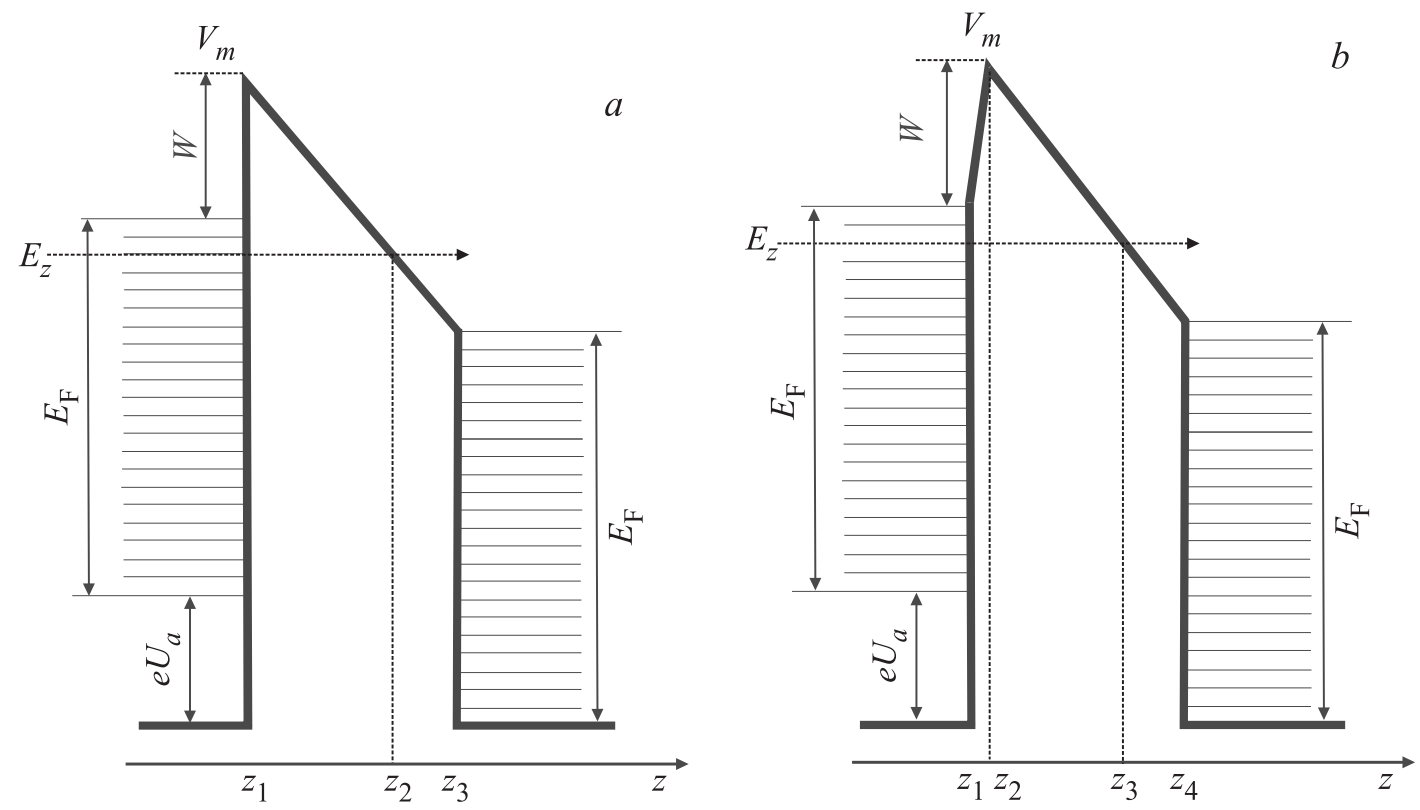

Рис. 4. Аппроксимация барьера трапецией с треугольником наверху $(a)$ и в виде двойного треугольного барьера $(b)$.

При $T>0$ можно положить $E_{m}^{+}=\infty$. Аппроксимируя $f(E)$ трапецией, спадающей от единицы до нуля в интервале $\mu-k T<E<\mu+k T$, получим при $k T \ll \mu$ результат

$$
\varphi\left(E_{z}\right)=\int_{0}^{\infty} f(E) d E_{\rho}=\mu-E_{z} .
$$

В общем случае этот интеграл Ферми-Дирака имеет вид

$$
\varphi\left(E_{z}\right)=k T \ln \left(1+\exp \left(\frac{\mu-E_{z}}{k T}\right)\right) .
$$

Если же ограничиться малыми температурами, получим

$$
\varphi\left(E_{z}\right)=\mu-E_{z}+k T \exp \left(-\frac{\mu-E_{z}}{k T}\right) .
$$

Для точного вычисления коэффициентов туннелирования $D_{i}^{ \pm}$следует решать уравнение Шредингера для соответствующего барьера. Это удобно делать, решая соответствующую задачу Коши одним из методов численного интегрирования обратным ходом, или заменяя барьер ступенчатым и используя метод матриц передачи. Для аналитического рассмотрения удобно использовать квазиклассическое (ВКБ) приближение. Самая простая модель соответствует $T=0$. В этом случае следует рассматривать барьеры для энергий $-E_{0}<E<-E_{0}+E_{F}$ при туннелировании с катода и для энергий $-E_{0}-e U_{a}<E<-E_{0}-e U_{a}+E_{F}$ при туннелировании с анода. В случае учета температуры на катоде $E_{m}<-E_{0}+E_{F}+k T$, а на аноде $E_{m}<-E_{0}-e U_{a}+E_{F}+k T$. В первом приближении форму барьера удобно аппроксимировать трапецией с треугольноком на верху рис. 4, $a$. Более точная аппроксимация - двойная трапеция рис. $4, b$ с формой в виде двойного треугольника для туннелирования с энергией Ферми. ВКБ приближение дает формулу для коэффициента туннелирования, обусловленного продольной частью кинетической энергии электрона $E_{z}$, в виде

$$
D\left(E_{z}\right)=\exp \left(-\frac{2 \sqrt{2 m}}{\hbar} \int_{z_{1}}^{z_{2}} \sqrt{V(z)-E_{z}} d z\right) .
$$

В этой формуле $t=z_{1}-z_{2}-$ ширина барьера на уровне $E_{z}$. Обычно нуль потенциальной энергии соответствует свободному электрону, удаленному в вакуум. При вычислении туннелирования удобно отсчитывать энергию от дна зоны проводимости на аноде. Тогда дно зоны проводимости на катоде имеет значение $e U_{a}$. Максимальную энергию электрона на катоде удобно взять в виде $E_{z m}=\mu+k T+e U_{a}$. Вероятность существования электрона с большей энергией экспоненциально мала. Наиболее вероятное туннелирование электрона с энергией чуть ниже уровня Ферми. Пусть $V_{m}$ - максимальная точка (высота) барьера. Ее можно взять в виде $V_{m}=e U_{a}+E_{F}+W$. Однако PB разная для различных барьеров. Удобно положить $z_{1}=0$ и определять толщину как функцию энергии: $t=t\left(E_{z}\right)$. Для треугольного барьера в (26) берем $V(z)=V_{m}\left(1-\alpha z / t_{0}\right)$ (рис. 4). Здесь $t_{0}-$ полная ширина барьера (на уровне Ферми): $t_{0}=z_{3}-z_{1}=z_{3} \approx d$, $\alpha=W / V_{m}$. Отсюда $t\left(E_{z}\right)=z_{2}=z_{3}\left(V_{m}-E_{z}\right) / E_{F}$. Заметим, что при $E_{z}<E_{F}$ следует брать $z_{2}=z_{3}$, т.е. всегда $z_{2}=z_{3}$. Поэтому туннелирование с анода идет через всю ширину барьера. Коэффициент такого обратного туннелирования экспоненциально меньше, чем прямого, 
поэтому при потенциале $e U_{a}$, сравнимом с энергией Ферми, обратный туннельный ток можно не учитывать. Отметим также, что при прямом туннелировании с верхних уровней энергия электронов диссипируется, что приводит к разогреву анода. При обратном туннелировании электроны получают энергию, и происходит охлаждение катода. Но этот эффект мал. Для трапецеидального (треугольного) барьера

$$
\begin{aligned}
& \int_{0}^{z_{2}} \sqrt{V_{m}-E_{z}-E_{F} z / z_{3}} d z \\
& \quad=\frac{2 z_{3}}{3 E_{F}}\left[\left(V_{m}-E_{z}\right)^{3 / 2}-\left(V_{m}-E_{z}-E_{F} z_{2} / z_{3}\right)^{3 / 2}\right] .
\end{aligned}
$$

Кроме трапецеидального барьера рис. 4, $a$ используем аппроксимацию в виде двух трапеций рис. 4, $b$. В области $0<z<z_{2}$ имеем $V(z)=V_{0}\left(1+V_{0}^{-1}\left(V_{m}-V_{0}\right) z / z_{2}\right)$, где $V_{0}=e U_{a}+E_{F}$. В области $z_{2}<z<z_{3}$ имеем $V(z)=V_{m}\left(1-\alpha\left(z-z_{2}\right) /\left(z_{4}-z_{2}\right)\right)$. Здесь $z_{4}=t_{0} \quad-$ полная ширина барьера, при этом также $z_{3}=z_{4}$, если $E_{z}<E_{F}$. Реально все параметры зависят от траектории $i$. Тогда в (26) имеем интеграл

$$
\begin{aligned}
\gamma_{i}\left(E_{z}\right)= & \int_{0}^{z_{3}} \sqrt{V_{i}(z)-E_{z}} d z=\int_{0}^{z_{2}} \sqrt{V_{i}(z)-E_{z}} d z \\
& +\int_{z_{2}}^{z_{3}} \sqrt{V_{i}(z)-E_{z}} d z
\end{aligned}
$$

для барьера вдоль траектории с номером $i$. Его вычисление дает значение

$$
\begin{aligned}
\gamma_{i} & =\frac{2 z_{2 i}}{3 W}\left[\left(V_{0}-E_{z}+W_{i}\right)^{3 / 2}-\left(V_{0}-E_{z}\right)^{3 / 2}\right]+\frac{2\left(z_{4 i}-z_{2 i}\right)}{3 E_{F}} \\
& \times\left[\left(V_{m i}-E_{z}\right)^{3 / 2}-\left(V_{m i}-E_{z}-\frac{E_{F} z_{3 i}}{z_{4 i}-z_{2 i}}\right)^{3 / 2}\right] \cdot \quad(27)
\end{aligned}
$$

Ширину барьера $z_{3}$ определяем из условия $E_{z}=V\left(z_{3}\right)$, откуда $z_{3}=z_{2}+\left(z_{4}-z_{2}\right)\left(V_{m}-E_{z}\right) / W$.

На рис. 5 приведены формы (профили) нескольких потенциальных барьеров, вычисленные при движении электрона вдоль соответствующих силовых линий (рис. 1). Наибольший туннельный ток дают барьеры с формой, соответствующей силовым линиям 4,5, проходящим через боковую поверхность вблизи угла. При учете действующих на заряд сил учтены плотности зарядов на поверхности и силы изображений, ближайших к заряду. На рис. 6 представлены теоретические результаты для усредненной по 36 траекториям в первом квадранте плотности тока

$$
J=\frac{1}{a^{2}} \sum_{i=1}^{N} J_{i} .
$$

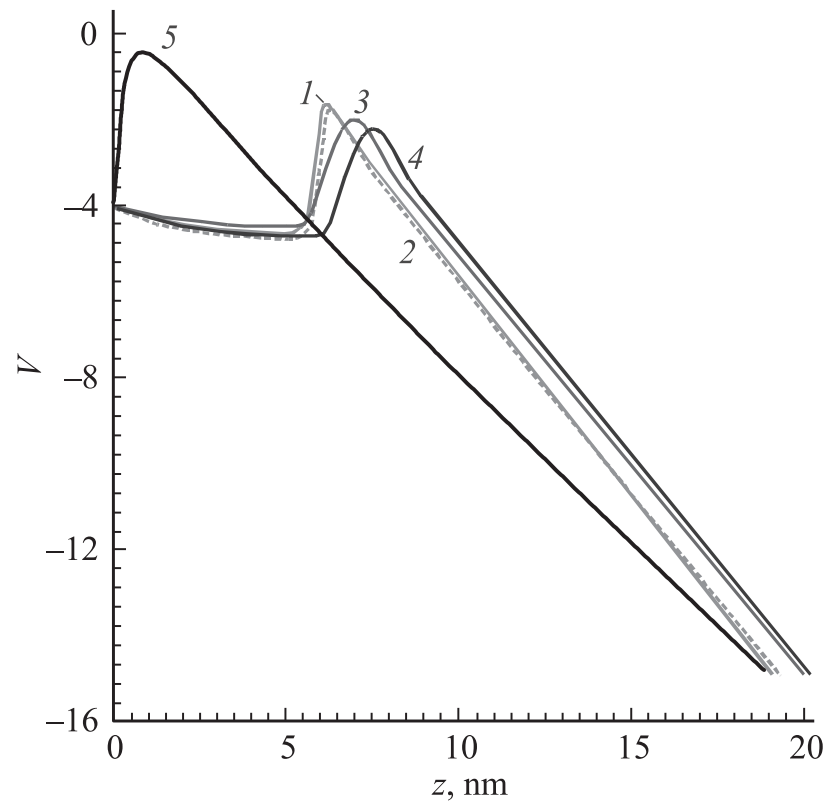

Рис. 5. Профили потенциальных барьеров (eV), вычисленные вдоль соответствующих силовых линий на рис. 1.

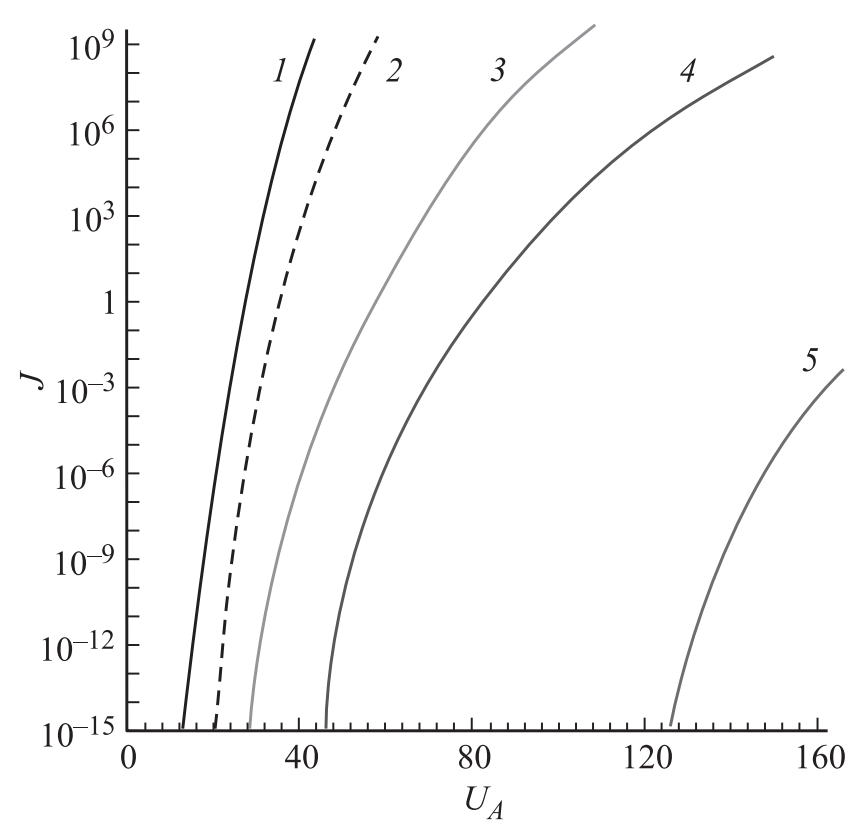

Рис. 6. Плотность туннельного тока $\left(\mathrm{A} / \mathrm{m}^{2}\right)$ в зависимости от напряжения на аноде (V) для диодных структур (рис. 1) с разными размерами промежутка $d: 20$ (кривая 1), 25 (2), $40(3), 60(4), 120(5) \mathrm{nm}$.

В случае $e U_{a} \gg E_{F}$ и больших $d$ форма барьера для туннелирования с катода хорошо соответствует треугольнику с линейным изменением ширины в зависимости от $E_{z}$, тогда как при туннелировании с анода ширина барьера постоянная, а форма приближенно описывается трапецией рис. 4, $a$. Более точно форма описывается двумя составленными трапециями рис. $4, b$. 
В случае $e U_{a} \ll E_{F}$ барьер близок к симметричному, описываемому двумя составленными трапециями. При вычислении (28) формы барьеров для траекторий определялись графически. Полные ширины барьеров $t_{0 i}$ несколько отличаются от $d$. Максимальное отличие (увеличение) имеет место в области граней и особенно углов. При этом в этих областях барьеры имеют существенно меньшую высоту, что говорит о повышенной эмиссии. Это также согласуется с эффектом усиления поля вблизи диэлектрического ребра. В области диэлектрика барьеры наиболее узкие, что также говорит о повышенной эмиссии через диэлектрические области по сравнению с эмиссией непосредственно с металла катода. Поэтому дополнительно целесообразно выполнить на катоде покрытие в виде пленки с высокой ДП, на которое наносить шахматную структуру из более низкой ДП. Перспективны также многослойные и градиентные покрытия с уменьшением ДП по направлению к аноду. В формуле (27) коэффициенты туннелирования вычислялись как $D_{i}=\exp \left(-2^{3 / 2} m^{1 / 2} \gamma_{i}\left(E_{z}\right)\right)$, при этом $\gamma_{i}$ определялись из (27), а $N_{i}^{ \pm}$из (23) при $E_{0}=e U_{z}$, $E_{m}^{-}=E_{F}, \varphi=E_{F}-E_{z}$, т. е. без учета температуры.

Экспериментально было изготовлено и исследовано несколько диодных структур с катодом из пластины кремния и нанесением на нее диэлектрической маски оксида кремния толщины $0.5 \mu \mathrm{m}$ с периодически вытравленными окнами размера $2 \times 2 \mu \mathrm{m} \mathrm{c} \mathrm{различными}$ концентрациями окон. Экспериментальное исследование эмиссии показало ее рост в три раза при концентрации окон 20000 на $\mathrm{mm}^{2}$ от уровня тока без травления окон, при этом плотность тока составила $10 \mathrm{~mA} / \mathrm{cm}^{2}$, а порог автоэмиссии падал от 74 до $50 \mathrm{~V} / \mu \mathrm{m}$. Экспериментальное исследование автоэмиссии в диодной структуре с наноразмерной пленкой из алмазографитовых кластеров (рис. 2) разной толщины приведено в [25]. Исследования выполнены при постоянном напряжении и расстоянии катод-анод $d=60 \mu \mathrm{m}$.

\section{Заключение}

В работе теоретически на основе классического подхода рассмотрено влияние на туннельный ток диэлектрических и полупроводниковых структур с низкой проводимостью и высокой диэлектрической проницаемостью, выполненных на проводящем катоде в плоской диодной структуре. Рассмотрены диэлектрические слои и периодические шахматные структуры. Теоретически и экспериментально показано влияние таких структур на холодную эмиссию, при этом сравнимая с толщиной барьера тонкая пленка с большой ДП существенно ее увеличивает, особенно при не очень больших по сравнению с ее толщиной расстояниях катод-анод. Это позволяет создавать симметричные относительно плоскости $z=0$ двусторонние эмиссионные структуры с наноразмерным расстоянием $d$. В этом случае вместо анода можно использовать сетку с потенциалом порядка десятков Вольт [2]. Образующийся двусторонний поток электронов можно повернуть на $90^{\circ}$, ускорить анодом и создать ленточный пучок с большим интегральным током [13]. Автоэмиссионная шахматная структура с выполнением периодических диэлектрических островков также демонстрирует увеличение тока эмиссии по сравнению с непокрытым катодом, а также и с полностью покрытым катодом. Это же относится к структуре с травлением окон в диэлектрической пленке в шахматном порядке. Имеет место максимум эмиссионной способности при некоторых оптимальных концентрациях окон или островков. Повышение эмиссии можно объяснить уменьшением ширин и высот потенциальных барьеров за счет влияния диэлектрика, увеличением эмиссионной поверхности путем создания пористой структуры и проникновением поля. Кроме этого, нанокластеры имеют характерные размеры в несколько $\mathrm{nm}$, создавая усиление поля и увеличивая поверхность эмиссии. С квантовой точки зрения можно говорить об увеличении числа поверхностных состояний и о проявлении размерных эффектов, учитывая размеры алмазографитовых кластеров. Технологически рассмотренные структуры перспективны для автоэмиссионных плоских панелей, а в случае структур из кремния для технологий на его основе.

Работа выполнена при финансовой поддержке гранта Российского научного фонда, проект № 16-19-10033.

\section{Список литературы}

[1] Fursey G.N. Field emission in vacuum micro-electronics, Kluwer Academic Plenum Publishers, NY: Springer, 2005. $205 \mathrm{p}$.

[2] Алехин Ю.В., Апин М.П., Буриев А.А., Бушуев Н.А., Воронков О.В., Григорьев Ю.А., Давидович М.В., Данилов А.Б., Исаев В.М., Кабанов И.Н., Кудряшов В.П., Куцько П.П., Мешанов В.П., Насенков И.Г., Рафалович А.Д., Роговин В.И., Рожнев А.Г., Шестеркин В.И. Сверхширокополосные лампы бегушей волны. Исследование в СВЧ-, КВЧ- и ТГЧ-диапазонах. Внедрение в производство / Под ред. Н.А. Бушуева. М.: Радиотехника, 2016. 480 с.

[3] Егоров Н.В., Шешин Е.П. Автоэлектронная эмиссия. Принципы и приборы. М.: Интеллект, 2011. 704 с.

[4] Проскуровский Д.И. Эмиссионная электроника. Томск: изд-во ТГУ, 2010. $288 \mathrm{c.}$

[5] Образиов А.Н., Павловский И.Ю., Волков А.П. // ЖТФ. 2001. Т. 71. Вып. 11. С. 89-95.

[6] Бобков А.Ф., Давыдов Е.В., Зайцев С.В., Карпов А.В., Козодаев М.А., Николаева И.Н., Попов М.О., Скороходов Е.Н., Суворов А.Л., Чеблуков Ю.Н. // ЖТФ. 2001. Т. 71. Вып. 6. С. 95-103.

[7] ЯФаров Р.К. // Известия вузов. Химия и химическая технология. 2016. Т. 59. № 8. С. 75-80.

[8] Ябаров Р.К., Горнев Е.С., Орлов С.Н., Тимошенков С.П., Тимошенков В.П., Тимошенков А.С. // Известия вузов. Электроника. 2016. Т. 21. № 2. С. 122-127.

[9] Фурсей Г.Н., Петрик В.И., Новиков Д.В. // ЖТФ. 2009. т. 79. Вып. 7. С. 122-126. 
[10] Фурсей Г.Н., Поляков М.А., Кантонистов А.А., Ябясов А.М., Павлов Б.С., Божевольнов В.Б. // ЖТФ. 2013. T. 83. Вып. 6. C. 71-77.

[11] Forbes R.G., Xanthakis J.P. // Surf. Interfac. Anal. 2007. Vol. 39. P. 139-145.

[12] Давидович М.В., Яфбаров Р.К. // Тр. Междунар. научно-техн. конф. и молодежной школы-семинара „Нанотехнологии-2010“. Таганрог: Изд-во Технологического ин-та ЮФУ, 2010. Т. 1. С. 52-54.

[13] Давидович М.В., Ябаров Р.К., Доронин Д.М. // Тр. 20-й Междунар. Крымской конф. СВЧ-техника и телекоммуникационные технологии (CriMiКo'2010). Севастополь, 2010. C. 733-734.

[14] Davidovich M.V., Bushuev N.A. // 2014. Tenth Intern. Vacuum Electron Sources Conference and Second International Conference on Emission Electronics. Saint-Petersburg: SaintPetersburg State University, 2014. P. 58-59.

[15] Davidovich M.V., Bushuev N.A., Yafarov R.K. // 2014. Tenth Intern. Vacuum Electron Sources Conference and Second International Conference on Emission Electronics. Saint-Petersburg: Saint-Petersburg State University, June 2014. P. 67-68.

[16] Давидович М.В., Бушуев Н.А. // Электроника и микроэлектроника СВЧ. 4-я Всерос. конф. СПб., СПбГЭТУ, 2015. T. 1. C. 245-249.

[17] Davidovich M.V., Bushuev N.A. // 2015. 25th Int. Crimean Conf. Microwave and Telecommunication Technology (CriMiCo'2015). Sevastopol: 2015. P. 145-146.

[18] Давидович М.В., Бушуев Н.А., Яфаров Р.К. // Актуальные проблемы электронного приборостроения. Материалы междун. научно-техн. конф. АПЭП’2016. СГТУ. 2016. Т. 1. C. 36-39.

[19] Давидович М.В., Бушуев Н.А., Ябаров Р.К. // Материалы 26-й Междун. научно-техн. конф. СВЧ-техника и телекоммуникационные технологии (КрыМиКо'2016). Севастополь: Вебер, 2016. С. 309-315.

[20] Simmons J.G. // J. Appl. Phys. 1963. Vol. 34. N 6. P. $1793-$ 1803.

[21] Simmons J.G. // J. Appl. Phys. 1963. Vol. 34. N 9. P. 25812590.

[22] Ахиезер А.И., Ахиезер И.А. Электромагнетизм и электромагнитные волны. М.: Высшая школа, 1985. 504 с.

[23] Тамм И., Блохинщев Д. // ЖЭТФ. Т. 3. Вып. 2. С. 77-100.

[24] Партенский М.Б. // УФН. 1979. Т. 128. С. 69-106.

[25] Яфаров Р.К. // ЖТФ. 2006. Т. 76. Вып. 1. С. 42-48. 